\title{
PERFIL SOCIOECONÔMICO, DEMOGRÁFICO E DE TRABALHO DOS AGENTES COMUNITÁRIOS DE SAÚDE
}

Mônica Motta Lino', Gabriela Marcellino de Melo Lanzoni', Gelson Luiz de Albuquerque², Mariana Cabral Schveitzer ${ }^{3}$

RESUMO: Estudo transversal e exploratório teve por objetivo caracterizar o perfil socioeconômico, demográfico e de trabalho dos Agentes Comunitários de Saúde de Florianópolis - Santa Catarina. Participou do estudo a população de 470 agentes, cujos dados foram coletados por instrumento estruturado e auto-aplicável. Identificou-se que os sujeitos possuem as seguintes características majoritariamente: mulher, branca, adulta jovem, casada, católica, com ensino médio completo, cuja ocupação prévia era exercida no comércio, natural e moradora da capital, mãe de um a dois filhos, cuja renda familiar mensal per capita média é de três salários mínimos e que atua na mesma equipe de saúde há cinco anos. Conclui-se que é possível vislumbrar as fortalezas e fragilidades deste grupo de trabalhadores para o melhor aproveitamento de seu potencial e melhorias no processo de trabalho, em busca da permanente qualificação das ações do Sistema Único de Saúde. PALAVRAS-CHAVE: Atenção primária à saúde; Programa saúde da família; Enfermagem.

\section{SOCIOECONOMIC, DEMOGRAPHIC AND WORK PROFILE OF COMMUNITY HEALTHCARE ASSISTANTS}

\begin{abstract}
This transversal and exploratory study aimed to characterize the socioeconomic, demographic and work profile of the community healthcare assistants in the city of Florianópolis in the state of Santa Catarina. 470 staff participated in the study, their details being collected by a structured, self-applied questionnaire. It was ascertained that the majority of the subjects had the following characteristics: female, Caucasian, young adult, married, Roman Catholic, educated to senior high school level, with previous work experience in commerce, both resident and born in the city, mother of one or two children, with an average per capita family income of three minimum salaries, and with five years' experience in the same healthcare team. It is concluded that it is possible to see the strengths and weaknesses of this group of workers, such that their potential may be maximized and the work processes improved, so as to continuously improve the standards of care given by the Brazilian National Health Service (Sistema Único de Saúde).
\end{abstract}

KEYWORDS: Primary healthcare; Family health program; Nursing.

\section{PERFIL SOCIOECONÓMICO, DEMOGRÁFICO Y DE TRABAJO DE LOS AGENTES COMUNITARIOS DE SALUD}

RESUMEN: Estudio transversal y exploratorio que tuvo por objetivo caracterizar el perfil socioeconómico, demográfico y de trabajo de los Agentes Comunitarios de salud de Florianópolis - Santa Catarina. Un total de 470 agentes participó del estudio, siendo sus datos recogidos por instrumento estructurado y autoaplicable. Se identificó que los sujetos poseen las siguientes características en su mayoría: mujer, blanca, adulta joven, casada, católica, con nivel medio completo, cuya ocupación previa era ejercida en el comercio, natural y viviendo en la capital, madre de un a dos hijos, cuya renta familiar mensal per capita media es de tres sueldos mínimos y que actua en el mismo equipo de salud por cinco años. Se concluye que es posible vislumbrar las fuerzas así como las fragilidades de este grupo de trabajadores para mejor aprovechar su potencial y mejorar el proceso de trabajo, en búsqueda de la permanente cualificación de las acciones del Sistema Único de Salud.

PALABRAS-CLAVE: Atención primaria a la salud ; Programa Salud de la Familia; Enfermería.

${ }^{1}$ Enfermeira. Doutoranda pelo Programa de Pós-Graduação em Enfermagem da Universidade Federal de Santa Catarina PEN UFSC. Bolsista CAPES.

${ }^{2}$ Enfermeiro. Doutor em Enfermagem. Professor do Departamento de Enfermagem e do PEN UFSC.

${ }^{3}$ Enfermeira. Doutoranda em Enfermagem pela Universidade de São Paulo.

Autor correspondente:

Recebido: 03/06/11

Mônica Motta Lino

Aprovado: $31 / 01 / 12$

Universidade Federal de Santa Catarina

Campus Universitário - 88040-970 - Florianópolis-SC-Brasil

E-mail: monicafloripa@hotmail.com 


\section{INTRODUÇÃO}

Diversos programas e políticas públicas vêm sendo estruturados a fim de fortalecer a implementação e demarcação do Sistema Único de Saúde (SUS) no Brasil. Dentre eles, anunciou-se a conformação de um programa com a atuação de Agentes Comunitários de Saúde (ACS) em municípios do Ceará para atuarem em regiões atingidas pela seca e com mortalidade infantil elevada, no ano 1991. Assim, iniciou-se o denominado Programa de Agentes Comunitários de Saúde (PACS) $)^{(1)}$, cujos trabalhadores são pessoas do povo que conhecem as necessidades de saúde da comunidade em que atuam e que podem auxiliar na organização e estruturação dos serviços de saúde em benefício coletivo, na atenção básica ${ }^{(2)}$.

Desde 1994, com a incorporação do Programa de Saúde da Família nos municípios brasileiros, posteriormente denominada Estratégia Saúde da Família (ESF), a Atenção Básica em Saúde adotou uma nova modelagem de atenção à saúde que preconiza ações de promoção da saúde, prevenção, recuperação, reabilitação de doenças e agravos mais frequentes e manutenção da saúde da comunidade, inserindo os trabalhadores de saúde no contexto de vida dos usuários e suas famílias ${ }^{(1)}$. Nesse cenário, as funções do ACS transcendem o campo da saúde, visto que requerem atenção a múltiplos aspectos das condições de vida da comunidade ${ }^{(3)}$. Entretanto, verificam-se na literatura científica alguns aspectos limitadores no trabalho do ACS ou motivos de insatisfação, como problemas com a própria saúde; falta de capacitação e de reconhecimento profissional $^{(4)}$ e diferença salarial ${ }^{(5)}$.

Para que se possa re-orientar e contribuir com o desenvolvimento da ESF e PACS, é fundamental conhecer quem são, quantos são e a qualificação profissional, tendo em vista que estes são aspectos inerentes ao processo de gestão do SUS nos municípios e contempla mudanças preconizadas desde a Reforma Sanitária brasileira. Assim, surge a indagação: Qual o perfil socioeconômico, demográfico e de trabalho dos Agentes Comunitários de Saúde no município de Florianópolis?

Esse estudo teve como objetivo caracterizar o perfil socioeconômico, demográfico e de trabalho dos ACS de Florianópolis/SC, uma vez que esta força de trabalho é considerável frente ao número total de profissionais de saúde e, em especial, porque este é o sujeito que tem chegado às residências com maior frequência. Portanto, retratá-los pode representar avanços na gestão de pessoas em saúde e, por conseguinte, na estruturação do próprio modelo.

\section{MÉTODO}

Trata-se de um estudo transversal, exploratóriodescritivo, uma vez que contribui com áreas onde a investigação não se tenha processado muito seguidamente, visto ser necessário desenvolver um processo de investigação que aponte a natureza do fenômeno e as características essenciais das variáveis que se pretende estudar $^{(6)}$. Assim, o objetivo de caracterizar o perfil socioeconômico, demográfico e de trabalho dos ACS coaduna-se com a pesquisa exploratório-descritiva, visto que explicita muitos fenômenos e variáveis que desta pesquisa poderão derivar.

A Secretaria Municipal de Saúde de Florianópolis possui em seu quadro de funcionários $516 \mathrm{ACS}$. Todos foram convidados a participar como sujeito de pesquisa e somente 46 ACS não foram incluídos na amostra final (9 estavam em férias no período de coleta de dados, 14 estavam em licença-saúde e 23 optaram por não participar da pesquisa). Assim, este estudo foi composto por $470 \operatorname{ACS}(95,33 \%)$, que estatisticamente representam a população destes trabalhadores. A coleta de dados ocorreu nos meses de abril, maio e junho do ano 2007, sendo utilizado para tanto, um instrumento estruturado, auto-aplicável. Foi realizada a validação do instrumento de pesquisa com um grupo piloto de ACS do município e profissionais do ramo da estatística. As variáveis selecionadas para a pesquisa foram: sexo, faixa etária, raça, religião, naturalidade, local de residência, local de trabalho, tempo de residência no município, tempo de residência no bairro, atividades exercidas para complementar a renda, estado civil, número de filhos, número de dependentes, renda familiar mensal per capita, tempo médio de trabalho como ACS, tempo de trabalho na Unidade Local de Saúde (ULS), atividades exercidas antes de ser ACS, escolaridade, se ainda estuda e, caso estude, em qual modalidade de ensino.

Com o suporte do Software EPIINFO ${ }^{\circledR}$ versão 6.0, que permite utilizar medidas de tendência central para avaliação das variáveis, assim como, medidas de avaliação de associação - testes estatísticos, os dados foram analisados a partir da prevalência das respostas registradas no Instrumento de Pesquisa para caracterizar os ACS. Assim, com a análise estatística descritiva, foram obtidos os registros das frequências e médias das variáveis encontradas.

Esta pesquisa foi aprovada no Comitê de Ética em Pesquisa com Seres Humanos da Universidade Federal de Santa Catarina-UFSC, sob número 049/2007. Foram respeitadas a resolução no 196/96 do Conselho Nacional de Saúde e as normas do principialismo bioético. 


\section{RESULTADOS}

Os ACS em exercício efetivo no município de Florianópolis eram predominantemente mulheres $(95,11 \%)$, brancas $(81,28 \%)$, católicas $(59,57 \%)$, com idade entre 28 e 47 anos, como pode ser visualizado na tabela 1.

Com relação aos dados socioeconômicos, verificase que os ACS são, em sua maioria, casados (62,13\%), possuem de um a dois filhos $(59,57 \%)$ /dependentes $(80,64 \%)$ e renda familiar mensal per capta de 1 a 3 salários. No que tange a escolaridade, observa-se que $65,11 \%$ dos ACS completaram o ensino médio e destaca-se que $8,08 \%$ estão cursando o ensino superior ou já o concluíram, como apresenta a tabela 2.

Quando questionados sobre a continuidade dos estudos, $24 \%$ dos ACS responderam que no momento estão estudando, enquanto $76 \%$ indicaram não estar estudando. Dentre os que ainda estão estudando, $16 \%$ afirmaram estar cursando o Ensino Médio, 7\% o Ensino Técnico, 6\% o Ensino Superior, 6\% em outros tipos de Ensino, 2\% o Ensino Fundamental e 63\% não responderam a questão. Entre aqueles que estão cursando o técnico ou superior, as indicações mais frequentes foram Curso Técnico em Enfermagem (47\%) e Magistério (8\%), seguido por superior em Pedagogia (9\%), Serviço Social (6\%), Teologia (3\%) e Outros com menos de $2 \%$ cada.

A naturalidade dos ACS é Florianópolis (73\%), seguido por Lages (7\%), pelo município de São José (5\%) que faz conurbação urbana com Florianópolis, a capital Porto Alegre (4\%), a capital São Paulo (3\%) e outras regiões ( $8 \%$ ), que englobaram diversas cidades do interior da Região Sul do Brasil.

Sobre o local de residência, $99 \%$ dos ACS indicaram residir em Florianópolis e 1\% respondeu que reside em outro município. No tocante se trabalha na comunidade onde mora, $95 \%$ respondeu que sim e 5\% respondeu que não. O gráfico 1 elucida sobre o tempo de residência dos ACS em Florianópolis e o tempo de residência no mesmo bairro.

Quando questionados sobre as atividades exercidas para complementar a renda, $13 \%$ dos ACS indicaram tra-

Tabela 1- Distribuição dos Agentes Comunitários de Saúde segundo características sócio-demográficas. Florianópolis, 2007

\begin{tabular}{lcc}
\hline \multicolumn{1}{c}{ Variáveis } & N & \% \\
\hline Sexo & 447 & 95,11 \\
Feminino & 23 & 4,89 \\
Masculino & & \\
Faixa etária (anos) & 80 & 17,02 \\
18 a 27 & 160 & 34,04 \\
28 a 37 & 139 & 29,57 \\
38 a 47 & 65 & 13,83 \\
48 a 57 & 26 & 5,53 \\
>7 & & \\
Raça & 5 & 1,06 \\
Amarelo & 382 & 81,28 \\
Branco & 2 & 0,43 \\
Indígena & 39 & 8,30 \\
Negro & 39 & 8,30 \\
Pardo & 3 & 0,64 \\
Não informado & & \\
Religião & 280 & 59,57 \\
Católico & 73 & 15,53 \\
Evangélico & 48 & 10,21 \\
Espírita & 11 & 2,34 \\
Testemunha de Jeová & 8 & 1,70 \\
Adventista & 9 & 1,91 \\
Umbandista & 2 & 0,43 \\
Outras religiões & 39 & 8,30 \\
Não tem religião & &
\end{tabular}


Tabela 2- Distribuição dos Agentes Comunitários de Saúde segundo características socioeconômicas. Florianópolis, 2007

\begin{tabular}{lcc}
\hline \multicolumn{1}{c}{ Variáveis } & N & \% \\
\hline Estado civil & 292 & 62,13 \\
Casado & 117 & 24,89 \\
Solteiro & 43 & 9,15 \\
Divorciado & 18 & 3,83 \\
Viúvo & & \\
Número de filhos & 142 & 30,21 \\
Um filho & 138 & 29,36 \\
Dois filhos & 92 & 19,57 \\
Três filhos & 42 & 8,94 \\
Quatro filhos ou mais & 56 & 11,91 \\
Não possui filhos & & \\
Número de dependentes & 283 & 60,21 \\
Uma a duas pessoas & 96 & 20,43 \\
Três ou mais pessoas & 91 & 19,36 \\
Não possui & & \\
Renda familiar mensal per capita (sm*) & 282 & 60,00 \\
R\$ 380,00 a R 1140,00 (1 a 3 salários) & 132 & 28,09 \\
R\$ 1141,00 a R\$ 1900,00 (3 a 5 salários) & 39 & 8,30 \\
R\$ 1901,00 a R 2660,00 (5 a 7 salários) & 17 & 3,62 \\
Acima de R 2660,00 (> 7 salários) & & \\
Escolaridade & 306 & 65,11 \\
Médio Completo & 55 & 11,70 \\
Fundamental Completo & 38 & 8,09 \\
Médio Incompleto & 19 & 4,04 \\
Superior Completo & 19 & 4,04 \\
Superior Incompleto & 19 & 4,04 \\
Fundamental Incompleto & 14 & 2,98 \\
Não Respondeu & & \\
\hline & &
\end{tabular}

*Base salário mínimo brasileiro R\$380,00 em 2007.

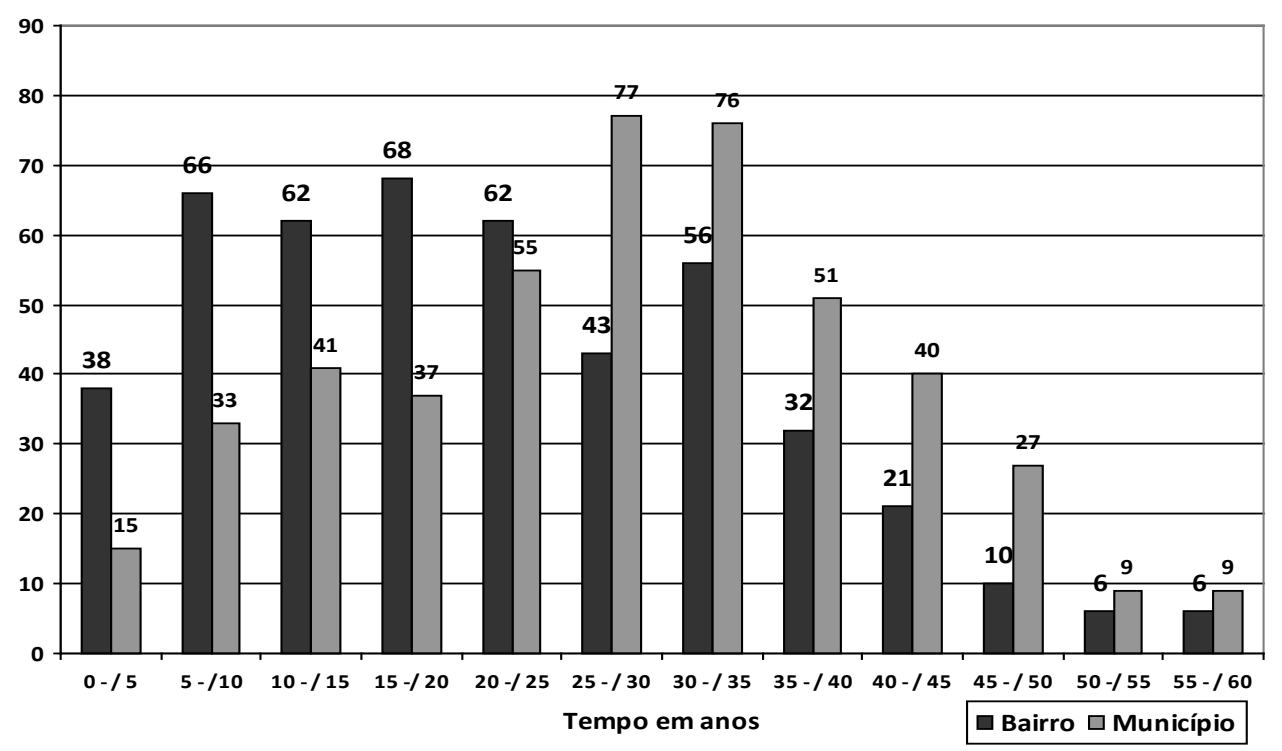

Gráfico 1- Distribuição dos Agentes Comunitários de Saúde segundo tempo de moradia no mesmo bairro e no mesmo município. Florianópolis, 2007. 
Tabela 3- Distribuição dos Agentes Comunitários de Saúde segundo atividades exercidas antes de ser ACS. Florianópolis, 2007

\begin{tabular}{lcc}
\hline \multicolumn{1}{c}{ Atividade } & N & \% \\
\hline Comércio e vendas & 354 & 75,32 \\
Serviços gerais & 93 & 19,79 \\
Secretária & 65 & 13,83 \\
Atividades na educação & 56 & 11,91 \\
Atividades na saúde & 40 & 8,51 \\
Atividades no ramo alimentício & 34 & 7,23 \\
Operadora de Telemarketing & 30 & 6,38 \\
Babá & 30 & 6,38 \\
Autônomo & 29 & 6,17 \\
Serviço de hotelaria & 29 & 6,17 \\
Costureira & 16 & 3,40 \\
Outros & 15 & 3,19 \\
Bancário & 12 & 2,55 \\
Dona de Casa & 9 & 1,91 \\
\end{tabular}

balhar em outro tipo de atividade remunerada, enquanto $86 \%$ não indicaram esta opção e $1 \%$ não respondeu a questão. Aqueles que responderam trabalhar em outro tipo de atividade remunerada citaram, atuar como vendedora de produtos por catálogo (32\%), desenvolver atividades na área da saúde - para aqueles que são profissionais técnicos ou auxiliares de enfermagem (18\%) $\mathrm{e}$, àqueles que recebem algum tipo de pensão (12\%).

O tempo médio de trabalho como ACS foi de cinco anos, que oscilou entre nove meses e 11 anos; enquanto o tempo médio de trabalho na ULS em que atua foi de quatro anos e oito meses. Na Tabela 3 são elucidadas as atividades exercidas antes de trabalharem como Agentes Comunitários de Saúde, com destaque para comércio e vendas $(75,32 \%)$.

\section{DISCUSSÃO}

Verificou-se que a população de ACS em Florianópolis era hegemonicamente do sexo feminino e sua faixa etária predominante oscilava entre 30 e 45 anos de idade, ou seja, composta por adultos jovens, o que é corroborada por pesquisa realizada em Santo André/SP( ${ }^{(7)}$. Esta realidade, ainda coincide com o perfil dos trabalhadores da saúde de outras regiões brasileiras, como a exemplo de Belo Horizonte, Distrito Federal, Porto Alegre, Recife, Salvador, São Paulo e Goiânia, onde a faixa etária predominante era acima dos 25 anos e a proporção feminina superava os $70 \%$ chegando a atingir $75,4 \%$ em Porto Alegre $^{(8-9)}$.

No setor organizativo em saúde, estudos evidenciam a predominância feminina compondo a força de trabalho, no entanto, a implicação desta questão de gênero necessita ser debatida de forma mais apurada ${ }^{(10)}$. A hipótese é a de que a escolha profissional da mulher é influenciada pela sua história familiar aliada ao contexto social que traz consigo, refletindo-se no exercício de "papéis femininos", como no caso da Enfermagem. A questão histórica justifica a atualidade, visto que o cuidado as pessoas era exercido apenas por mulheres religiosas na Idade Moderna. Posteriormente, houve o aproveitamento da mão de obra feminina no decorrer da Revolução Industrial e, atualmente, evidencia-se a permanência da "dupla jornada de trabalho", na qual a mulher necessita conciliar o trabalho profissional com o trabalho doméstico e o cuidado à família ${ }^{(11)}$.

Assim como as lutas feministas por conquistas sociais nas questões de gênero ${ }^{(11)}$, existem também articulações em outras categorias no tocante a relação de poder, como é o caso da distribuição de trabalhadores segundo raça. Os ACS em Florianópolis declaram-se, em sua grande maioria, como de raça branca, corroborando aos índices nacionais que agregam baixa representatividade da população negra no setor da saúde ${ }^{(8)}$. No entanto, esta proporcionalidade se deve, em grande parte, ao fato da colonização na região ser de origem européia, sendo consequência histórica natural o maior número de pessoas de raça branca na população total residente em Florianópolis, o que não acarreta influências significativas ao processo de trabalho do ACS neste tocante. Cumpre destacar que esta colonização justifica a adoção do catolicismo pela maioria (91\%) dos ACS, como herança de imigrantes advindos de países essencialmente católicos. Em Porto Alegre, a questão da religiosidade dos ACS mostra si- 
milaridades: 92\% dos ACS adotam alguma religião e, corroborando, a hegemonia é da Igreja Católica ${ }^{(9)}$.

Nos últimos anos, a questão sobre religiosidade/ espiritualidade tem demonstrado impacto sobre a saúde física das pessoas, sendo possível fator de prevenção de doenças e outros agravos em populações previamente sadias. Segundo a literatura, quem adota alguma religião tem melhor índice de atividade imunológica, menor índice de mortalidade e morbidade, redução de cardiopatias, de hipertensão arterial e de depressão $0^{(12,13)}$. Além disso, a questão da religiosidade tem todo um contexto histórico-social que permite ao ACS maior interação junto à comunidade em que atua.

A religiosidade e a saúde são estruturas conceituais que se entrelaçam, e que, nesse aspecto podemos "perceber até que ponto a multiplicidade de sistemas religiosos que permeia o universo cultural dos urbanos pobres, oferecendo distintas visões de mundo, oferecem possibilidade não apenas circunscritas ao encontro momentâneo de cura das aflições no campo da Saúde, mas conformam novos valores e padrões comportamentais que, de forma duradoura, são incorporados pelos indivíduos, implicando numa "domesticação" do corpo com consequências para a saúde ${ }^{(14)}$. Nesse sentido, o ACS, católico na sua maioria, deverá perceber a importância de ser e agir dentro da multiplicidade de credos, convicções e crenças religiosas nas quais o seu trabalho está envolvido.

No que tange ao relacionamento interpessoal e vínculo com a comunidade, os ACS são os únicos trabalhadores da equipe de saúde da Atenção Básica que devem residir no local em que atuam por no mínimo dois anos. Cumprindo com as exigências estabelecidas pelo Ministério da Saúde ${ }^{(15)}$, a maioria dos ACS de Florianópolis reside na mesma comunidade por um período entre 20 a 25 anos, sendo que o tempo médio de atuação na mesma área de abrangência é de cinco anos. Verifica-se na literatura que há alta rotatividade de ACS em outras regiões do país e é possível encontrar trabalhadores com dificuldade de compreensão das funções que lhes são atribuídas ${ }^{(5)}$.

Certamente, a média elevada de atuação na mesma unidade de saúde e moradia no próprio local de trabalho, podem influenciar, positivamente, na articulação do serviço com a comunidade e no enfrentamento da complexidade do trabalho em saúde. Ainda que o tempo de residência possa ser um fator que influencie a qualidade da relação entre agente e comunidade, pois as pessoas o consideram um vizinho antes mesmo de ACS, visto que frequentam os mesmos espaços sociais e têm acesso à sua casa, há que se considerar outros aspectos importantes, como a empatia, para a integração desse trabalhador com os usuários ${ }^{(9)}$.

Outra característica fundamental para atuar como ACS é ter facilidade para se comunicar, visto que atua como interlocutor entre unidade de saúde e comunidade realizando visitas domiciliares e atividades coletivas de educação e saúde ${ }^{(5)}$. Foi verificado que a grande maioria dos ACS de Florianópolis já possuía experiência com atendimento ao público, executando atividades como vendedor, balconista, secretária, caixa de supermercado, auxiliar de sala, professor, e aproximadamente $10 \%$ dos trabalhadores já desempenharam atividades na área da saúde, a saber: auxiliares de consultório odontológico, auxiliares de enfermagem e técnicos em Enfermagem. Aproveitando esta característica, os serviços de saúde poderiam investir mais nestes trabalhadores para que apoiassem programas de educação sanitária.

Assim, visando a selecionar trabalhadores com o perfil adequado ao município de Florianópolis, como em todo território nacional, não foi estabelecido uma única forma de contratação no início da incorporação dos ACS ao SUS. No ano de 2002, os ACS tornaram-se uma categoria profissional por meio da Lei Federal n. 10.507 de 10 de julho de 2002, sendo atualmente regidos pela Lei n. 11.350, de 5 de outubro de 2006, a qual regulamenta a contratação através de concurso público ou de forma terceirizada, sob o regime da CLT ${ }^{(16)}$.

Embora as dificuldades referentes às formas de contratação tenham sido superadas, a remuneração do ACS ainda é considerada baixa ${ }^{(5,8,17)}$, com reflexo direto na satisfação do trabalhador que se sente pouco valorizado $^{(5)}$. Nesta pesquisa, $10 \%$ destes trabalhadores assumiu desempenhar outras atividades fora do horário de trabalho para complementar a renda familiar. Esse fator ganha destaque como fragilidade para a atuação destes sujeitos, tendo em vista seu consequente reflexo na satisfação em relação ao trabalho, sua interferência no comprometimento com as ações laborais desenvolvidas, bem como no investimento em atividades de lazer, esportivas e educacionais.

Esta necessidade emerge a partir do contexto doméstico dos ACS, os quais são na sua maioria mães, casadas, possuidoras de um filho ou dois dependentes. Assim, as exigências da mulher contemporânea é que, além de ter um emprego que auxilia na renda familiar, também desenvolva atividades domésticas em sua própria residência, cuide dos filhos e do marido, restando pouco tempo para o cuidado de $\mathrm{si}^{(18)}$.

A combinação desses aspectos, principalmente o 
econômico, configura-se como um obstáculo quando os ACS almejam investir, por exemplo, nos estudos para aplicação na Equipe de Saúde da Família. Assim, como resposta às alterações na regulamentação da atividade do ACS em 2006, as quais passaram a exigir ensino fundamental completo, bem como, a conclusão de um curso de qualificação básica ${ }^{(18)}$, verifica-se que estes trabalhadores têm buscado qualificar-se concluindo o ensino fundamental e médio, realizando cursos profissionalizantes na área da saúde, bem como, cursos superiores. Neste sentido, estudos ${ }^{(7-8)}$ revelam esta tendência em diversas regiões do país, sinalizando que, além da necessidade para a contratação, a elevação do nível de escolaridade vai ao encontro do aumento dos desafios encontrados na sociedade e a ampliação das atividades dos ACS com a ESF. Considera-se positiva a elevação do nível de escolaridade dos ACS de Florianópolis, visto que seu reflexo pode favorecer os processos interacionais com a comunidade e equipe, bem como, ampliar a criticidade e criatividade do trabalhador.

Configuram-se como limitações deste estudo a coleta dos dados referente à 2007 e a não realização de correlações entre as variáveis selecionadas. Entretanto, foi possível atingir os objetivos propostos e conhecer de forma mais abrangente quem são os ACS que atuam em Florianópolis. Destaca-se o desafio na elaboração deste estudo pela proposta ousada, na medida em que poucos trabalhos desta natureza foram levados a efeito. Como potencialidades, indicam-se a possibilidade de melhor compreensão sobre os ACS e sua atuação na comunidade.

\section{CONCLUSÃO}

Enquanto os demais profissionais da equipe de saúde já possuem posições e espaços consolidados, o ACS está construindo sua identidade e estruturando sua conduta como membros de uma equipe multiprofissional. Conhecido o perfil socioeconômico, demográfico e de trabalho do ACS e suas principais características, é possível identificar as fortalezas e fragilidades deste grupo de trabalhadores para o melhor aproveitamento de seu potencial e melhorias em seu processo de trabalho, para qualificar e capilarizar as ações do Sistema Único de Saúde.

Os resultados do presente trabalho justificam repensar as formas de participação dos ACS de Florianópolis na ESF e os programas de formação, visto que estes trabalhadores são, em sua maioria, pessoas interessadas em investir em sua formação e grandes conhecedores da comunidade onde atuam, pois residem nela há muitos anos.

\section{REFERÊNCIAS}

1. Ministério da Saúde (BR). Programa de Agente Comunitário de Saúde - PACS. Brasília (DF): Ministério da Saúde; 2001.

2. Silva JA, Dalmaso ASW. O agente comunitário de saúde e suas atribuições: os desafios para os processos de formação de recursos humanos em saúde. Interface. 2002;6(10):75-96.

3. Nogueira PR, Silva RBFZA. A vinculação institucional de um trabalhador sui generis - o agente comunitário de saúde. 2000. [acesso em 11 mar 2011]. Disponível: http://www.ipea.gov.br/pub/ td/td_2000/td0735.pdf

4. Trindade L, Peron A, Amestoy S, Gehlen G, Noguez P. Reflections on the shape of attendance in the family health strategy. Cogitare enferm. 2011;16(1):162-6.

5. Santos KT, Saliba NA, Moimaz SAS, Arcieri RM, Carvalho ML. Community health agent: status adapted with family health program reality? Cienc. saude colet. 2011;16(Supl. 1):1023-8.

6. Köche JC. Fundamentos de metodologia científica: teoria da ciência e prática da pesquisa. $26^{\mathrm{a}}$ ed. Petrópolis (SP): Vozes; 2008.

7. Pessoni A. O perfil dos agentes comunitários do programa saúde da família e a forma de capacitação empregada no treinamento destes profissionais: estudo de caso em Santo André. In: Anais da Conferência Brasileira de Comunicação e Saúde. 2001 [acesso em 10 mar 2011]. Disponível: http://www.projetoradix.com. br/arq_artigo/IV_05.pdf

8. Cotta RMM, Schott M, Azeredo CM, Franceschini SCC, Priore SE, Dias G. Organização do trabalho e perfil dos profissionais do Programa Saúde da Família: um desafio na reestruturação da atenção básica em saúde. Epidemiol. serv. saude. 2006;15(3):7-18.

9. Ferraz L, Aerts DRGC. O cotidiano de trabalho do agente comunitário de saúde no PSF em Porto Alegre. Cienc. saude colet. 2005;10(2):347-55.

10. Cecílio LCO. Gênero e estudos organizacionais: apontamentos para futuros estudos. Cienc. saude colet. 2009;14(4):107-8. 
11. Padilha MICS, Vaghetti HH, Brodersen G. Gênero e enfermagem: uma análise reflexiva. Rev. enferm. UERJ. 2006;14(2):292-300.

12. Guimarães HP, Avezum A. O impacto da espiritualidade na saúde física. Rev. psiquiatr. clín. 2007;34(1):88-94.

13. Lucchetti G, Lucchetti ALG, Avezum Junior A. Religiosity, spirituality and cardiovascular disease. Rev. bras. cardiol. 2011; 24(1):55-7.

14. Couto MT. Religiosidade, reprodução e saúde em famílias urbanas pobres. Interface Comunic saúde educ. 2001;5(8):27-44.

15. Brasil. Portaria n. 64 de 28 de março de 2006. Diretrizes e normas para a organização da Atenção Básica para o Programa Saúde da Família (PSF) e o Programa Agentes Comunitários de Saúde (PACS). Diário Oficial da União 2006; 28 mar.

16. Brasil. Lei n. 11.350 de 5 de outubro de 2006. Define que as atividades de Agente Comunitário de Saúde e de Agente de Combate às Endemias. Diário Oficial da União. Brasília, 2006.

17. Santana JB, Vasconcelos A, Martins C, Barros J, Soares J, Dutra B. Agente comunitário de saúde: percepções na estratégia saúde da família. Cogitare enferm. 2009;14(4):645-52.

18. Lunardi VL, Lunardi WD Filho, Silveira RS, Soares NV, Lipinski JM. Self care as a condition for care to others in health practice. Rev. latino-am. enfermagem. 2004;12(6):933-9. 